\title{
Temporal Patterns of Muscle Activation for Arm Movements in Three-dimensional Space
}

\author{
Martha Flanders \\ Department of Physiology, University of Minnesota, Minneapolis, Minnesota 55455
}

\begin{abstract}
Little is known about the patterns of muscle activation that subserve arm movement in three-dimensional space. In this study, activation patterns of seven arm muscles were related to the spatial direction of human arm movement. Twenty movement directions defined two orthogonal vertical planes in space. The arm movements were moderately paced; each movement lasted approximately $\mathbf{5 0 0}$ msec. New techniques of EMG analysis were developed to describe the temporal pattern of muscle activation. For each muscle, a principal component analysis revealed a common phasic and tonic waveform for all directions of movement, within one plane. A temporal shifting procedure based on best covariance values revealed activation delays associated with different movement directions. The results show a consistent pattern of temporal shifting of the common waveform for movements in different directions. Coupled with past results showing that activation amplitude is a function of the cosine angle of movement or force direction, the present results suggest a relatively simple control strategy for mechanically complex arm movements: neural circuits produce a common phasic and tonic activation waveform that is scaled in amplitude and delayed in time, depending on the desired movement direction.
\end{abstract}

We "think" about moving our arms in terms of the spatial characteristics of the intended movement: populations of neurons in the primate brain encode parameters related to kinematics (spatial position and its derivatives). Georgopoulos and co-workers (Gcorgopoulos ct al., 1983, 1988; Georgopoulos and Massey, 1988) have shown that the direction of an arm movement can be predicted by quantifying the activity of populations of neurons in the motor cortex. Others have made similar observations in several movement-related brain areas (Fortier et al., 1989; Alexander and Crutcher, 1990; Kalaska et al., 1990). The possibility of a partial encoding of kinetic (force) parameters in some of these brain areas has also been explored (Kalaska et al., 1989). However, the form of the neural transformation from kinematic (or kinetic) representations in the brain to patterns of motoneuronal activity in the spinal cord is unknown.

Very little is known about the patterns of muscle activation subserving arm movements in three-dimensional space. To a first approximation, the total amount (average amplitude across time) of activity in a given muscle varies with the cosine angle

\footnotetext{
Received Jan. 18, 1991; revised Apr. 9, 1991; accepted Apr. 10, 1991.

This work was supported by NIH Grant R01 NS-27484. I thank Dr. John F. Soechting.

Correspondence should be addressed to Martha Flanders, Department of Physiology, 6-255 Millard Hall, University of Minnesota, Minneapolis, MN 55455.

Copyright $@ 1991$ Society for Neuroscience $0270-6474 / 91 / 112680-14 \$ 03.00 / 0$
}

of the movement direction (Georgopoulos et al., 1984b). Cosine "tuning curves" can be constructed to relate the total amount of activation to the direction of movement, just as these tuning curves have been constructed to relate cortical activity to movement direction (Georgopoulos et al., 1983) and to relate muscle activity to static force direction (Flanders and Soechting, 1990). Cortical tuning curves are generally unimodal, but muscle tuning curves are often more complex. Muscle tuning curves are often bimodal, showing one cosine peak in directions for which the muscle is an agonist (producing a positive contribution to the net force) and a separate cosine tuning function in directions for which the muscle is an antagonist (producing a negative contribution to the net force).

Studies of single-joint movements or multijoint movements in the horizontal plane have revealed an involvement of antagonist muscles in shaping the temporal profile of an arm movement. The studies from many laboratories (reviewed in Discussion) have shown that targeted movements performed as quickly as possible (e.g., 200-msec movement time) are subserved by a "triphasic" pattern of muscle activation. Ideally, the initiation of a distinct burst of activity in the agonist is followed by a distinct burst of activity in the antagonist. After a brief silent period in the agonist, activity returns in the form of a second burst (or a more prolonged activation if the new posture requires it). In most cases, these studies use horizontal movements starting from rest and supported against gravity, so that muscle activity shows a clear onset and offset. For movements that are not supported against gravity and are not performed as quickly as possible, it is often impossible to identify the onset or termination of bursts of EMG activity.

This study combines and extends previous work on triphasic temporal patterns and on directional tuning curves by relating temporal patterns of muscle activation to the direction of movement in three-dimensional space. We used 20 movement directions in two vertical planes (sagittal and frontal planes). We used movements of moderate speed (500-msec movement time) and developed a technique for EMG analysis that did not depend on the delineation of bursts.

In spite of the apparent complexity of our approach (compared to past approaches), we were able to identify a simple algorithm by which neural networks generate activation patterns for spatial movements. The data reveal that there is a basic waveform of muscle activation that is scaled in amplitude and shifted in time to generate movements in different directions.

\section{Materials and Methods \\ Experimental design and apparatus}

The experiment was designed to vary only one kinematic parameter, while keeping all other kinematic parameters relatively constant. Thus, 

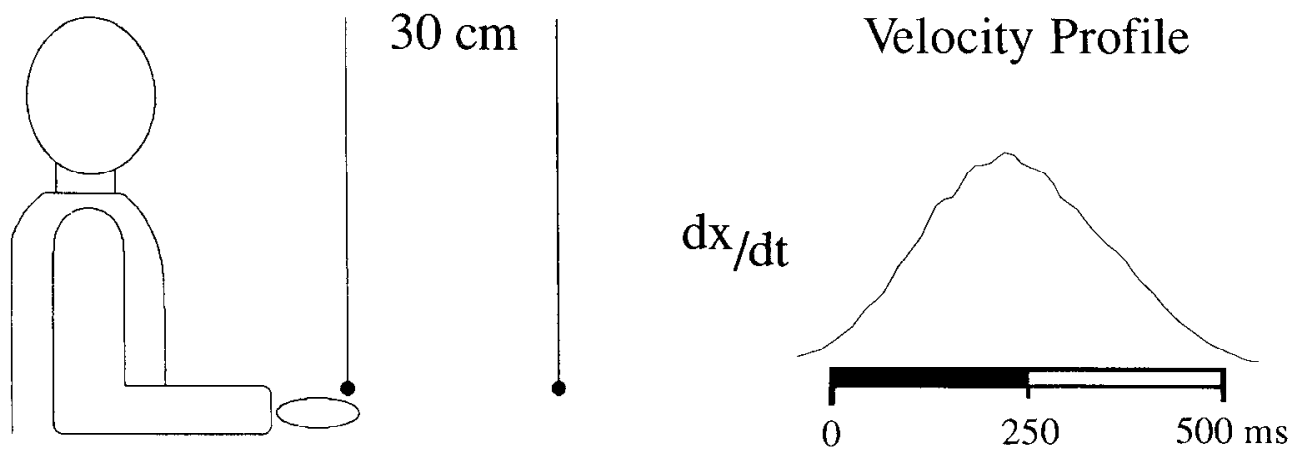

Figure 1. Kinematics. The experiment was designed to keep the initial arm position, the movement amplitude, the velocity profile, and the movement time relatively constant. The initial arm position and the movement amplitude of $30 \mathrm{~cm}$ are shown schematically on the left. The single-peaked velocity profile (right) is the average of the first derivatives of five horizontal path records (data from subject B). The black and white time bar will be used in subsequent figures to mark movement onset and the approximate movement time of $500 \mathrm{msec}$.

the subjects were trained to produce movements in 20 different directions, with a relatively constant initial position, amplitude, movement time, and velocity profile. The kinematics of the hand movement were recorded using an ultrasonic device (Graph Pen GP-8-3D) interfaced to a computer (Macintosh IIx). Hand position in three dimensions was recorded with a spatial resolution of $0.1 \mathrm{~mm}$ and a temporal resolution of $10 \mathrm{msec}(100 \mathrm{~Hz})$.

The kinematic features of the movements are shown in Figures 1 and 2. Figure 1 (left) shows schematically the initial arm posture and the movement amplitude. Human subjects stood erect with the upper arm vertical (shoulder relaxed) and the forearm horizontal (elbow flexed), in a sagittal plane. Deviations from this initial posture would be expected to cause differences in arm muscle activation patterns (Flanders and Soechting, 1990), and so we attempted to keep the initial posture constant throughout the experiments. For each movement, both the initial and the final (target) hand positions were marked by sinkers suspended from the ceiling with fishing line. The target direction illustrated in Figure 1 is straight forward, in the sagittal plane. Other directions were indicated by hanging the target in different locations. The required movement amplitude was always $30 \mathrm{~cm}$.

Target directions were spaced $36^{\circ}$ apart, as indicated by the records of hand path in Figure 2. We trained subjects to move from the initial position to the target as consistently as possible. The subject moved to the same target on five consecutive trails (the five path records for each direction in Fig. 2). The "go" signal was a computer-generated tone; it was followed at a constant interval by a second tone. The velocity profile was displayed after each trial, and trials with "nonstandard" velocity characteristics were discarded and repeated. Nonstandard movements could also be discarded after the experiment, upon closer inspection in subsequent analysis.

A "standard" velocity profile is shown in Figure 1 (right). This sym- metric profile is an average of the differentiated horizontal path signal $(d x / d t)$ for five movements to the right in the frontal plane (the movemcnts labcled "lateral" in Fig. 2). Wc also accepted asymmctric velocity profiles in our analysis (the degree of symmetry varied with movement direction). However, we retained and analyzed only movements with a single velocity peak and a movement time of approximately $500 \mathrm{msec}$ (400-600 msec was acceptable). Because the focus of this study was the relationship between muscle activation and required movement direction, we did not systematically analyze deviations from a symmetric velocity profile and a straight path. We also did not temporally scale the EMG data to account for deviations from a $500-\mathrm{msec}$ movement time.

Three normal subjects took part in this experiment: a $5^{\prime} 9^{\prime \prime}, 130-1 \mathrm{~b}$ female (subject A), a 5'10", 150-lb male (subject B), and a 6 $6^{\prime \prime}, 190-\mathrm{lb}$ male (subject $\mathrm{C}$ ). The basic experiment consisted of five consecutive movements in each of 20 directions, at moderate speed (as shown in Figs. 1,2). This experiment was run twice on each of the three subjects. Subjects A and B also performed the $5 \times 20$ movements as fast as possible, for comparison with the moderately paced movements. Results from the three subjects were very similar, and in most cases, we will show data from whichever subject exhibited a particular phenomenon most clearly.

\section{Electromyographic data analysis}

Acquisition. We used small bipolar surface electrodes (2-mm diameter) to record the activity of superficial arm muscles. The electrodes were placed about $2 \mathrm{~cm}$ apart over the bellies (or middle portions) of each muscle. We will report the patterns recorded from the following muscles: (1) anterior deltoid (AD), clavicle - lateral humerus (origin - insertion); (2) pectoralis ( $\mathrm{Pec}$ ), clavicle and sternum - lateral humerus; (3) posterior

\section{Sagittal Plane}

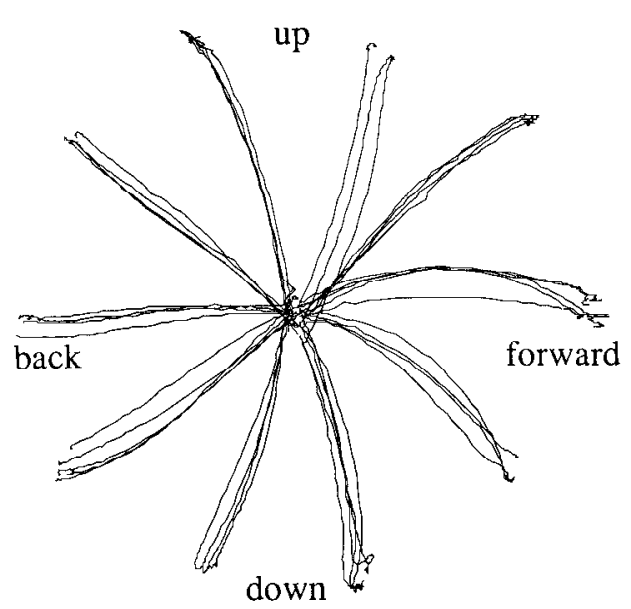

\section{Frontal Plane}

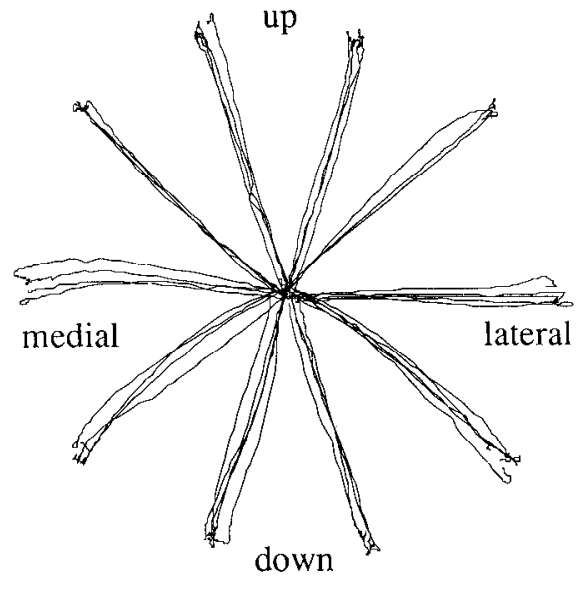

Figure 2. Hand paths for the moderately paced arm movements of subject B. The subject moved to the same target in five consecutive trials to achieve the most consistent performance possible. The movement amplitude was always $30 \mathrm{~cm}$. Four of these path directions are for targets in the horizontal plane: the paths labeled back and forward in the sagittal plane (left) and the paths labeled medial and lateral in the frontal plane (right). 
deltoid (PD), scapular spine - lateral humerus; (4) medial deltoid (MD), acromion of the scapula - lateral humerus; (b) biceps (BI), scapula radius (crossing both the shoulder and the elbow); (6) brachioradialis (BR), lateral humerus-radius; and (7) medial triceps (MT), posterior humerus-ulna. We also recorded activity from the latissimus dorsi and from the long head of triceps, but we will not show these data. The activity of the latissimus dorsi resembled the activity of the posterior deltoid, but the latissimus dorsi was typically active in only two or three of the 10 directions in each plane, giving an insufficient sample. The EMG recorded from the long head of triceps was not consistent across the three subjects, and we suspected a recording artifact. In subject $B$, Pec showed very little activity (perhaps due to a bad electrode contact), and for this muscle we will only report on subjects $A$ and $C$.

The EMG signals were preamplified $(1000 \times)$, band-pass filtered $(100-$ $5000 \mathrm{~Hz}$ ), and then amplified again $(10 \times)$ prior to digitization. The data were digitized at $500 \mathrm{~Hz}$. For some experiments, the data were digitally high-pass filtered ( $100-\mathrm{Hz}$ cutoff) to remove low-frequency noise. After amplification, filtering, and digitization, the data were rectified.

Averaging and normalization. We prepared the EMG records for further analysis using the following four steps.

(1) We aligned three to five like records (same direction) at the onset of the velocity profile and averaged. The onset of the velocity profile was defined as the point at which the velocity record rose above the amplitude of the baseline signal and was measured by hand for each trial, using a computer cursor program. We truncated the EMG record (originally $1 \mathrm{sec}$ long) to include $100 \mathrm{msec}$ before velocity onset and $600 \mathrm{msec}$ after velocity onset. Thus, the subsequent analyses were based on 700-msec-long, averaged EMG records.

(2) We resampled the averaged EMG records by taking the average amplitude of 10 -msec-long bins. We will refer to these resampled, averaged EMG records as "traces."

(3) Because we wished to focus on the temporal profile of the EMG waveform, rather than on the total amplitude, we normalized for amplitude. For each trace, we subtracted the average amplitude of the trace and divided by the root mean square (RMS) of the amplitude (as we will illustrate in Results and in Fig. 4). If, for example, a trace were sinusoidal, this procedure would set its baseline to zero and normalize its peak-to-peak amplitude with respect to other traces.

(4) Not all muscles were active for all directions of movement. For directions in which the RMS amplitude did not exceed a predetcrmincd value (based on noise level), the trace was discarded from further analysis. Thus, 5-10 traces were used for each analysis.

For each muscle, we did a separate analysis on the data from each of the three subjects, in each of the two planes, and in each of the two repetitions of the basic experiment. Thus, for each muscle, we performed the temporal shifting and principal component analysis (described below) 12 times.

Temporal shifting. After averaging and normalization, we computed the dot products of pairs of traces and used these covariance values to compute the temporal shift that best aligned the EMG waveforms (the dot product is the sum of the sample-by-sample products). Within each group of 5-10 traces, we chose the trace with the largest RMS value as a template upon which to align the other traces. We then took pairs containing the largest trace and a sccond trace and computed the covariance for each of 20 possible temporal realignments. We shifted (realigned) the second trace by as much as $100 \mathrm{msec}$ earlier or $100 \mathrm{msec}$ later, in 10-msec increments. The appropriate temporal realignment was chosen as the temporal shift giving the largest absolute value of covariance (the maximum or minimum of the cross-correlation function). Thus, if the largest covariance was negative, the realignment would be based on a negative fit (a reciprocal pattern).

Principal component analysis. We used a principal component (PC) analysis to quantify the waveforms of the rectified EMG signals. This statistical technique is becoming a popular tool in neurophysiology, and several detailed accounts of the method have been published (Richmond and Optican, 1987; Osborn and Poppele, 1989; Soechting and Lacquaniti, 1989). PC analysis is useful in quantifying the "shape" of electrophysiological waveforms.

In general, $\mathrm{PC}$ analysis transforms a set of data waveforms into a set of PC waveforms, which are derived from the original data. PC analysis is similar to Fourier analysis in that, after the transformation, any individual data waveform can be reconstructed as a weighted sum of the $\mathrm{PC}$ waveforms. However, unlike Fourier analysis, in which the weighted sum is composed of sinusoidal waveforms, the shapes of the PC waveforms are chosen in light of the data waveforms.
By definition, the first principal component (PC1) contributes most prominently to the reconstruction of the set of data waveforms (the components are ranked according to their relative contributions). The PCl resembles the data most closely and can be thought of as an "average" of the data waveforms. However, PCl is not a conventional "average" because it can be derived from both positive and negative versions of the data waveforms. Therefore, as will be shown in Results, PC1 should sometimes be viewed as if on a reversed (upside-down) amplitude scale.

A set of $10 \mathrm{EMG}$ waveforms transforms into $10 \mathrm{PC}$ waveforms. These were calculated by first constructing a symmetric matrix containing the covariance values of pairs of normalized EMG traces. We then computed the 10 eigenvalues $(\lambda)$ of this matrix and their corresponding eigenvectors (u). Each eigenvalue corresponded to a PC, and we ranked the $\lambda$-PC pair according to the magnitude of $\lambda$. The PCs were computed as

$$
\mathrm{PC}_{n}(t)=\left(1 / \sqrt{\lambda_{n}}\right) \sum u_{m n} \mathrm{EMG}_{m}(t),
$$

where $n$ is the rank (1-10) of the PC, and the summation is over the products of the first through tenth EMG waveforms and their corresponding first through tenth elements of the eigenvector ( $m$ index). Both the PC and the EMG waveforms are functions of time $(t)$.

The basic formula for reconstruction of a data waveform from the principal components is

$$
\mathrm{FMG}_{m}(t)=\sum\left(\sqrt{\lambda_{n}}\right) u_{m n} \mathrm{PC}_{n}(t),
$$

where the product $(\sqrt{\lambda}) u$ is the "weighting coefficient" for each PC, and the summation is over the 10 weighting coefficients and their corresponding PCs ( $n$ index). This formula would reconstruct the RMSnormalized EMG waveforms that were input to the PC analysis. Therefore, because we normalized prior to the analysis by dividing by RMS amplitude, in Results we will report weighting coefficients that have been remultiplied by the RMS value for each direction.

The percentage of the variance of the set accounted for by the first principal component is given by

$$
\lambda_{1} / \sqrt{\Sigma}\left(\lambda_{n}{ }^{2}\right) \text {. }
$$

Comparisons between the relative contributions of the second and first PCs will be made by giving the ratio of the associated eigenvalues $\left(\lambda_{2} /\right.$ $\lambda_{1}$ ).

\section{Results}

\section{Anterior deltoid}

In this section, we will use data from the AD to illustrate qualitatively the directional tuning of rectified, averaged EMG records and to compare these records to the normalized traces used in the subsequent analyses. We will then present the central result of this study: a basic waveform can be shifted in time to correspond to movements in different directions. We will also show cases in which the basic waveform gives a negative (reciprocal) fit to the data in directions for which the $A D$ is an antagonist.

Directional tuning of EMG amplitude. For movements in the sagittal plane, the overall amplitude of activity in the AD is a unimodal function of movement direction. In Figure 3, we show rectified, averaged EMG records for the 10 directions of movement in the sagittal plane (subject A). The time bar under each record marks the movement onset and indicates that the movement time was approximately $500 \mathrm{msec}$ (see Materials and Methods). The amplitude scale is arbitrary, but uniform. The EMG records are laid out in a way that corresponds to the handpath data shown in Figure 2 (left). The layout also corresponds to the hand-centered polar plots used in a previous study (inset). In the previous study (Flanders and Soechting, 1990), we modeled EMG amplitude (the distance from the center of the plot to the data point) as a cosine function(s) of static force direction. The inset shows the static tuning curve for subject A's AD. This muscle was most active when the arm produced static forces 


\section{Anterior Deltoid Sagittal Plane}
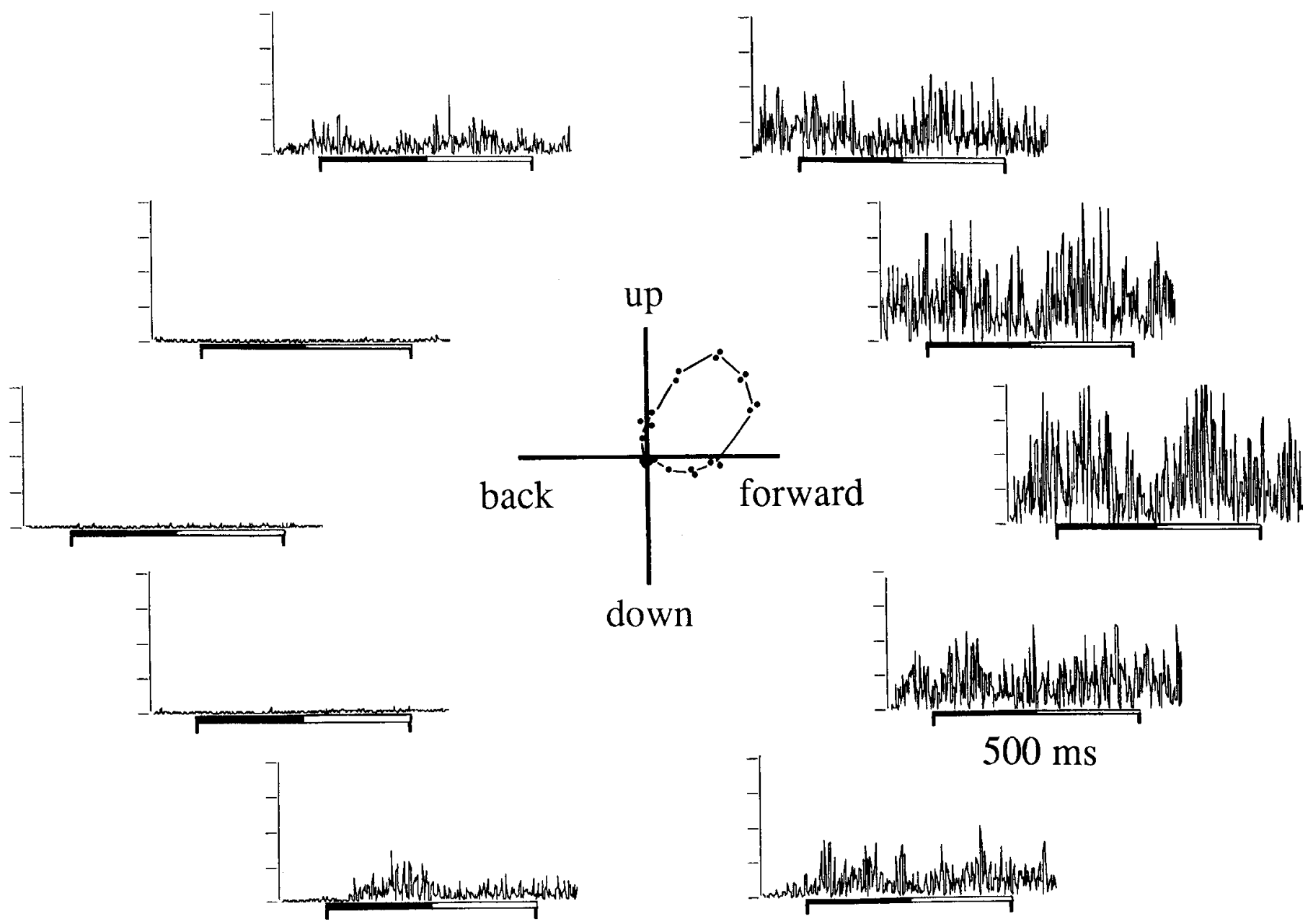

Figure 3. Rectified and averaged EMG records from subject A's AD, for movements in 10 directions (indicated by the layout) in the sagittal plane. This muscle was not active for the three backward movement directions. The largest RMS activation was for the forward direction, and the earliest activation onset (above noise level) was for the forward/slightly upward direction, where the muscle became active more than 100 msec prior to movement onset. The movement times are indicated by the black and white time bars. The inset shows a static tuning curve for this same muscle. These inset data were obtained in a previous study (Flanders and Soechting, 1990) and are shown for comparison with the dynamic amplitude tuning.

upward and forward. The best directions (largest amplitude) for the static and dynamic conditions do not exactly coincide. However, the overall form of the dynamic amplitude tuning corresponds to the static tuning: the AD was less active as the movement direction deviated from the best direction.

The directional tuning of this particular muscle is unimodal: unlike many other muscles, there is no significant activity for the direction opposite the best direction. However, inhomogeneous temporal characteristics occur within the directional range of this unimodal tuning curve. For movements up and slightly back, the onset of activation is prior to the onset of movement, whereas for movements down and slightly back, activation appears to reach threshold well after movement onset. For movement directions in between these two extremes, there appears to be a continuous shift in the timing of activation.

This type of temporal shift is also apparent in Figure 4, in which we show EMG records from the same AD, for hand-path directions in the frontal plane. In this plane, $A D$ is an agonist for movements upward and medial, and the activity for these directions is large and early. For downward and lateral movements, the records show what might be called an "antagonist burst" (cf. Gottlieb et al., 1989). In these latter directions, AD is mechanically an antagonist, and the timing of the activity appears to be appropriate for braking the movement.

The inset of Figure 4 illustrates the convention we adopted for processing these EMG records prior to covariance calculations. The data from medial and medial/slightly downward directions are shown smoothed by averaging in 10 -msec bins. The amplitude scale has been shifted to contain positive and negative values around a zero baseline, and the amplitude has been normalized for RMS (see Materials and Methods). The temporal waveform is seen more clearly as a result of these manipulations.

Quantification of the temporal shift. A qualitative inspection of Figures 3 and 4 suggests that these data share a common waveform. Although the activations start at different times relative to the onset of the movement, each record shows a curve 


\section{Anterior Deltoid Frontal Plane}
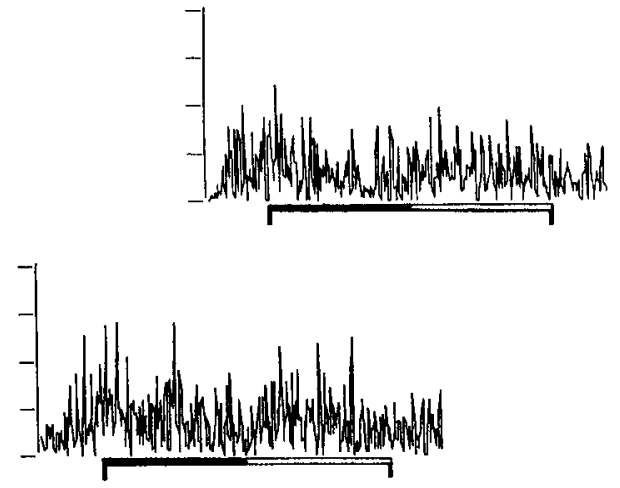
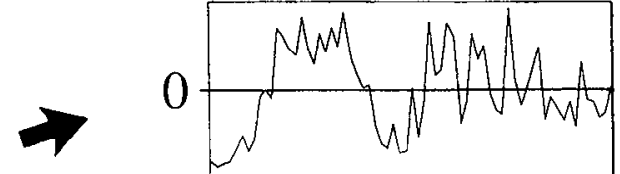

0
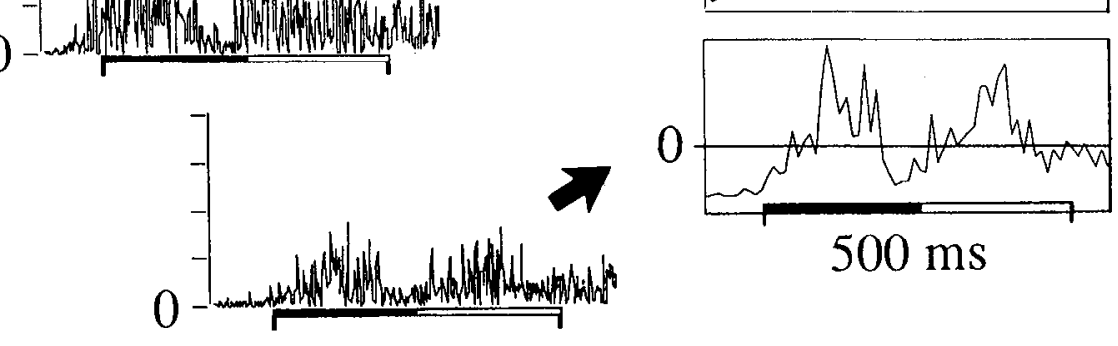
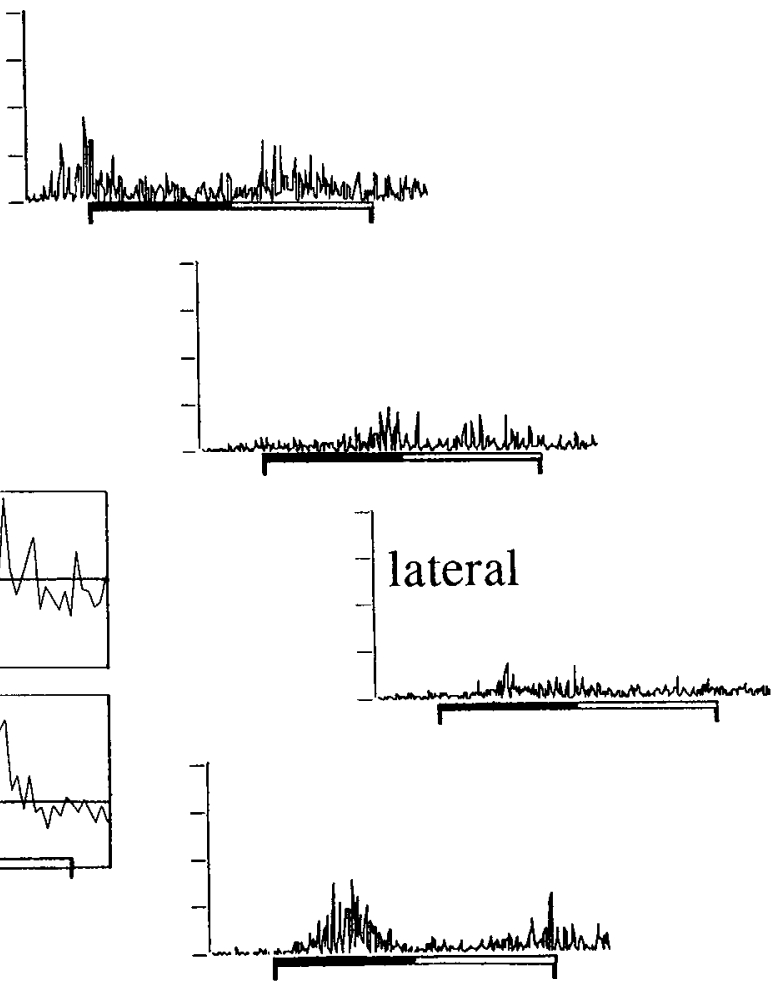

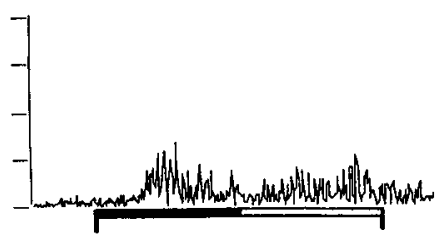

down

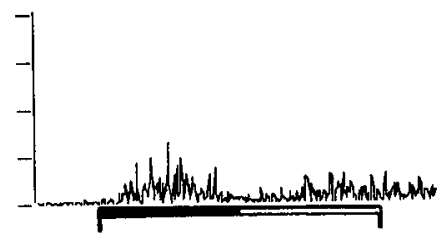

Figure 4. Rectified and averaged EMG records from subject A's AD for movements in the frontal plane. The inset shows the smoothing and normalization procedure. Consistent with this inset, in subsequent figures a single tick mark on the amplitude scale will represent the 0 baseline.

of activation-inactivation-activation and then a more sustained period of activation: a "phasic and tonic" pattern. We used a temporal shifting procedure coupled with a PC analysis to quantify the temporal position of a common waveform in traces from various directions.

In Figure 5, we illustrate the use of this procedure on $\mathrm{AD}$ traces for movement in the sagittal plane (from the data shown in Fig. 3). Figure $5 A$ shows the $\mathrm{PCl}$ calculated from traces in the same time frame (aligned on movement onset). This PCl does not adequately represent the set of data waveforms from which it is derived; it accounts for only $64 \%$ of the variance of the set. This PCl begins with a relatively square pulse of activation that does not resemble the phasic curve seen in the EMG records in Figure 3. This is because the timing of the phasic portion varies so as to obscure its shape in the PC. The inactivation after this square pulse occurs as a result of the fact that the EMG records for the two downward directions were fit with a negative version of $\mathrm{PCl}$ (see Materials and Methods).

In Figure $5 B$, we show the temporal shift performed to align the EMG waveforms prior to recalculation of PC1. By convention, we adopted the scale shown in the inset of Figure 5 to plot these temporal-shift data as a function of movement direction. Movements straight forward had the largest RMS amplitude and thus served as the template for realignment of the other traces. Traces for movement straight forward and forward/slightly upward required no realignment and thus appear at the positive covariance zero mark, two-thirds of the way out from the center of the polar plot (broken line). Directions for which traces were shifted later in time (up to $-100 \mathrm{msec}$ ) have data points farther out on the polar plot. For the upward/slightly backward and upward/slightly forward directions, the EMG waveform occurred earlier and therefore had to be shifted later in time by $-70 \mathrm{msec}$ or $-20 \mathrm{msec}$, respectively. For downward movements, the waveform was delayed and therefore had to be shifted earlier in time for the best alignment with the template.

In Figure 5C, we show the $\mathrm{PC} 1$ calculated after realigning the traces. This second $\mathrm{PC}$ analysis revealed a waveform that more closely resembled the phasic and tonic pattern observed in the EMG records (cf. Fig. 3). After realignment, the traces from all directions were fit with a positive version of this $\mathrm{PCl}$. This $\mathrm{PCl}$ accounted for $76 \%$ of the variance of the set, a $12 \%$ improvement over the $\mathrm{PCl}$ calculated prior to realignment. Thus, the temporal 


\section{Anterior Deltoid Sagittal Plane}

A) Before Temporal Shift

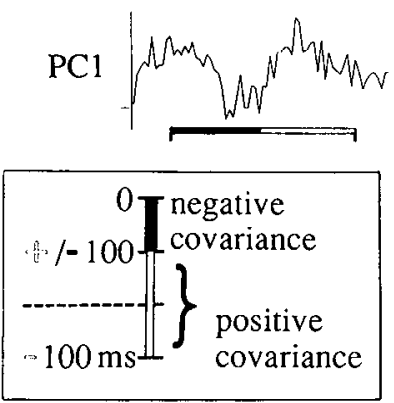

B) Temporal Shift

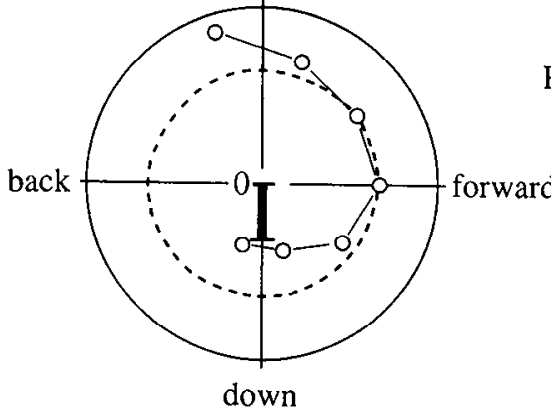

C) After Temporal Shift

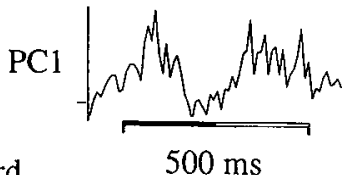

Figure 5. The PCl of AD before $(A)$ and after $(C)$ the temporal shifting procedure. In $B$, we use a polar plot of movement direction to show the amount of temporal shift needed to best realign the smoothed and normalized EMG traces. The scale for this polar plot is shown in the inset: there is a black region of negative covariance that ranges from 0 to $-100 \mathrm{msec}$ temporal shift and a white region of positive covariance that ranges from +100 to -100 msec temporal shift. By convention, we have equated a -100 -msec (later) shift for a negative fit with a $+100-$ msec (earlier) shift for a positive fit. Both the broken line and the origin represent zero temporal shift. The black bar on the polar plot shows the region for a negative fit. Subject $A$, sagittal plane. shifting operation allowed the calculation of a $\mathrm{PCl}$ waveform that resembled the data waveforms much more closely than did the $\mathrm{PCl}$ calculated prior to realignment.

The temporal shift plots for the other two subjects were very similar to the plot for subject A. In Figure 6, we compare the plot previously shown in Figure 5 to the corresponding plots for the other two subjects. The temporal shift plot for subject $B$ is nearly identical to the plot for subject A. Subject C shows a similar pattern, except that the largest covariance for the upward/slightly backward direction was negative (solid circle). This trace was shifted later in time for a negative (reciprocal) fit to the template. The occurrence of large negative covariance values was prominent in other muscles, as well as in the frontal plane data for AD.

Traces with negative covariance. In Figure 7, we show that negative covariance was a prominent feature of $\mathrm{AD}$ activity for directions in the frontal plane. In Figure $7 A$, we show the $\mathrm{AD}$ temporal shift plot for the frontal plane. As in subsequent figures, we used the same method and the same scale as illustrated in Figure 5. The largest RMS activity was in the medial/slightly upward direction. Thus, the temporal shift for this direction is zero. There is relatively little temporal shifting for directions on either side of this template. In directions opposite the template, there was also relatively little temporal shifting, as indicated by the fact that data are at or near zero. In directions opposite the largest RMS activity, however, the traces had a negative covariance (solid circles).

In Figure $7 B$, we show the $\mathrm{PCl}$ waveform calculated after the temporal realignment reported in Figure $7 A$. This $\mathrm{PCl}$ accounted for $89 \%$ of the variance in the set. Although the temporal shifting plot for the frontal plane is different from the plot for the sagittal plane (cf. Fig. 6), the PCl waveform for the frontal plane shows a very similar phasic and tonic pattern (cf. Fig. 5C). Thus, this basic AD waveform appears to be similar across all directions of movement in three-dimensional space.

In Figure $7 C$, we show the $\mathrm{PCl}$ weighting coefficients on a polar plot of movement direction. For each direction, the distance from the center of the plot to the data point represents the absolute magnitude of the $\mathrm{PCl}$ weighting coefficient ( $\times$ RMS) needed to reconstruct the trace. Positive weighting coefficients are indicated by the open squares, whereas negative weighting coefficients are indicated by the solid squares. Negative weighting coefficients occur in the region for which $\mathrm{AD}$ is an antagonist and correspond to the negative covariance values (solid circles)

\section{Anterior Deltoid Sagittal Plane}
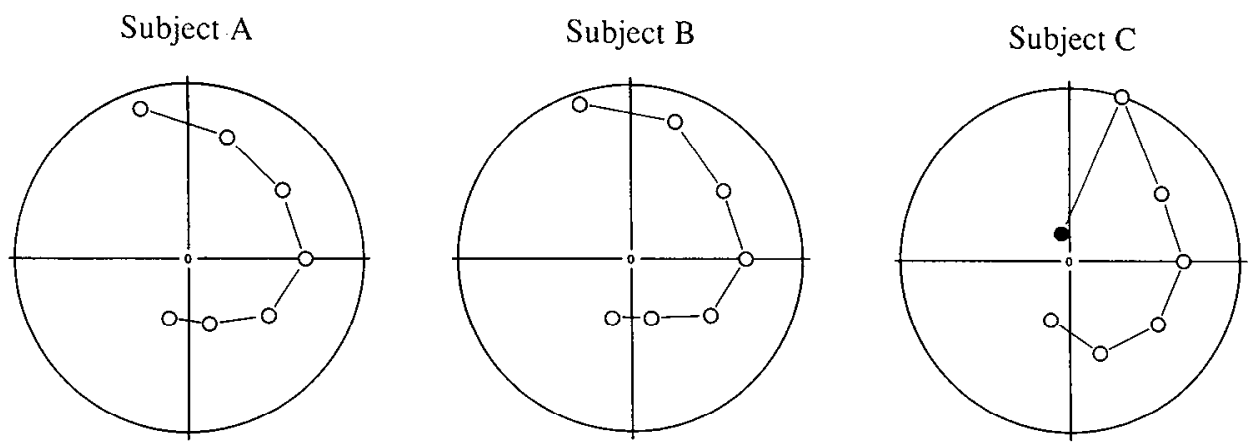

back $\rightarrow$ forward

Figure 6. Temporal shift pattern. The form of the temporal shift plot for $A D$ was nearly uniform across the three subjects. The data for subject $A$ are repeated from Figure 5 for the sake of comparison with the corresponding data from the other two subjects. Subject $C$ shows a negative fit for upward/slightly backward movements (solid circle). AD, sagittal plane. The scale is as in Figure 5 . 


\section{Anterior Deltoid Frontal Plane}

A) Temporal Shift
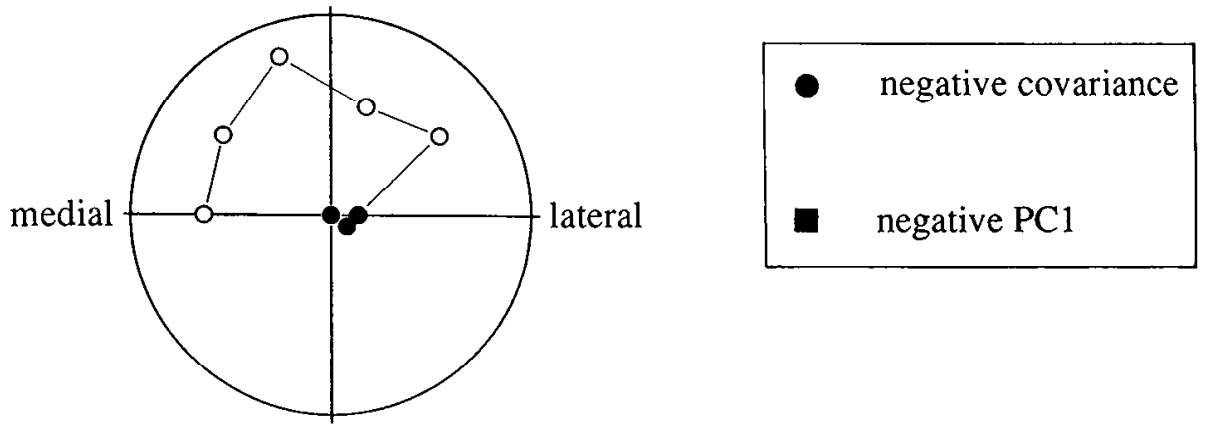

Figure 7. Reciprocal pattern. For directions where the muscle is mechanically an antagonist, EMG traces were often given a negative fit. Frontal plane data from subject C's AD show a temporal shift plot with a reciprocal region $(A$, solid circles), a phasic and tonic $\mathrm{PC1}$ waveform $(B)$, and a polar plot of $\mathrm{PCl}$ weighting coefficients $(\times$ RMS amplitude) with a reciprocal region $(C$, solid squares). The temporal shift scale is as in Figure 5.
B) PC1 Waveform

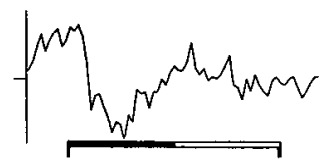

shown in Figure $7 A$. For movement downward/slightly lateral, the large negative weighting coefficient indicates that the trace closely resembles an upside-down version of $\mathrm{PC} 1$.

\section{Pectoralis and $\mathrm{PC} 2$}

In the previous section, we presented data regarding only the first $\mathrm{PC}$ of $\mathrm{AD}$. To reconstruct a data waveform completely, it is necessary to use all of the PCs. In this section, we use data from Pec to show a second principal component (PC2), and data from all muscles to report the relative importance of PC2.

Figure $8 A$ shows the $\mathrm{PC} 1$ waveform and the $\mathrm{PC1}$ weighting coefficients ( $\times$ RMS) for directions in the sagittal plane (square symbols). The weighting coefficients exhibit a bimodal tuning function similar to that reported in our previous study for isometric contractions of this muscle (Flanders and Soechting, 1990). Positive weighting coefficients correspond to upward movements, whereas negative weighting coefficients correspond to downward movements. The PCl waveform resembles the phasic and tonic pattern, though the phasic inactivation is not as prominent as it is for $\mathrm{AD}$. This waveform accounted for $74 \%$ of the variance in the set.

Figure $8 B$ shows that the PC2 has a faster phasic waveform. This quick burst of activation-inactivation is followed by a much more gradual increase in activation. This waveform accounted for $42 \%$ of the variance in the set. (Thus, PC1 and PC2 together accounted for $85 \%$ of the variance).

The distribution of PC2 weighting coefficients (triangular symbols) is quite different from the distribution of the $\mathrm{PCl}$ weighting coefficients. In Figure $8 B$, the scale on the weighting coefficient plot is arbitrary, but identical to the scale used in
Figure $8 A$ for the $\mathrm{PC} 1$. Thus, it is possible to compare directly the relative contributions of $\mathrm{PCl}$ and $\mathrm{PC} 2$ for each direction. For the forward direction, the faster phasic pattern prevailed.

The PC1, by definition, describes the entire set of data better than the set is described by any of the other components. However, the example shown above suggests that PC2 is important in providing a more complete picture of the activation pattern. The relative importance of $\mathrm{PC} 2$ in describing Pec activation, however, was not typical of most muscles.

For most muscles, $\mathrm{PC} 2$ made a relatively small contribution to the reconstruction of data waveforms. The relative contributions of the PCs are quantified using the ratio of the eigenvalues associated with each PC (see Materials and Methods). For the example shown in Figure 8, the ratio of the PC2 eigenvalue to the PCl eigenvalue was 0.57 . In other muscles, this ratio was usually well below $1 / 2$. Typical values for elbow muscles (MT, BR, and BI) were about $1 / 4$. For MD, typical ratios were about $1 / 3$. In these muscles, the PC2 waveform showed no clear temporal pattern and was not qualitatively repeatable either within or across subjects.

In $\mathrm{AD}$ and $\mathrm{PD}$, the ratios were somewhat higher, but as in most muscles, the $\mathrm{PC} 2$ waveforms were not qualitatively repeatable. In $A D$, the ratios were slightly higher in the sagittal plane than in the frontal plane, and these ratios were repeatable both within and across subjects (sagittal plane: subject $\mathrm{A}, 0.48$ and 0.56 ; subject $B, 0.48$ and 0.55 ; subject $C, 0.46$ and 0.50 ; frontal plane: subject $A, 0.35$ and 0.39 ; subject $B, 0.44$ and 0.46 ; subject $C, 0.26$ and 0.31 ). In $P D$, the ratios ranged from 0.31 to 0.71 (mean, 0.51 ), and the distribution of ratios across planes and across subjects was less orderly than for AD. 


\section{Pectoralis Sagittal Plane}

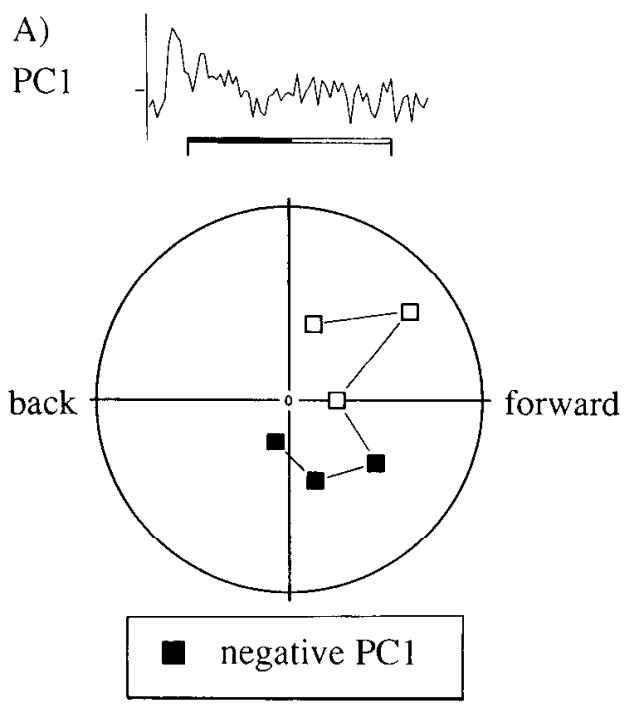

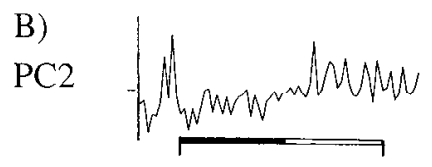

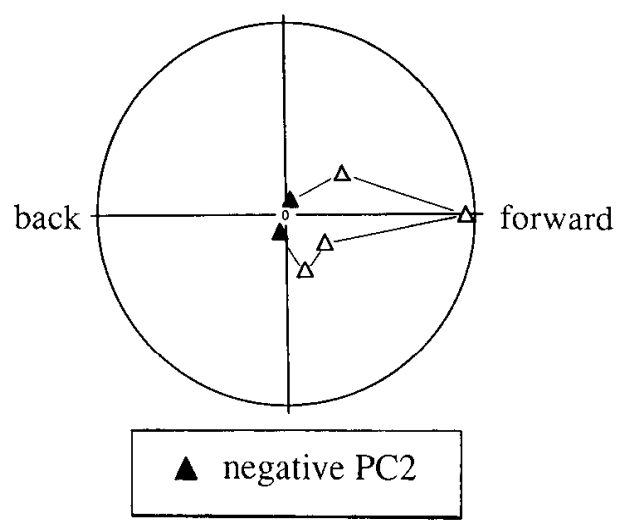

Figure 8. Pec was the exception to our general observation that higher-order PCs usually represented random variability. For movement in the sagittal plane, $\mathrm{PC1}$ weighting coefficients had a bimodal distribution ( $A$, squares), whereas PC2 weighting coefficients had a unimodal distribution that complemented the $\mathrm{PCl}$ distribution $(B$, triangles). For forward movements, a faster phasic waveform (PC2) predominated the EMG. The weighting coefficient scale is arbitrary but the same in $A$ and $B$. Subject A.

\section{Posterior and medial deltoid}

Data from PD and MD show the same sort of temporal shift plot and PC1 waveform as exhibited by AD. In this section, we will show the temporal shifts associated with a phasic and tonic PD waveform for movements in both planes and a phasic and tonic $\mathrm{MD}$ waveform for movements in the frontal plane. We will also show a less phasic MD waveform for movement in the sagittal plane.

Frontal plane. In Figure 9, we show the temporal shifts and $\mathrm{PCl}$ waveforms for $\mathrm{PD}(A)$ and $\mathrm{MD}(B)$. The temporal shifting patterns are basically similar to those shown for AD. They exhibit both a continuous curve of positive covariance (open circles) and a region of negative covariance (solid circles).

For PD, the phasic and tonic PC1 waveform was typically seen in all three subjects, in all six experiments. In the example shown in Figure $9 A$, this waveform accounted for $79 \%$ of the variance.
For $\mathrm{MD}$, the initial phasic portion of the $\mathrm{PC} 1$ waveform was brief. The example shown in Figure $9 B$ accounted for $89 \%$ of the variance of the set. In one of the six repetitions of the experiment, the initial phasic portion was nearly absent. Without this initial burst, the waveform resembled the more tonic waveform that we will show below for MD activation in the sagittal plane.

Sagittal plane. For movement in the sagittal planc, PD showcd an abrupt transition between a positive and negative $\mathrm{PCl}$ waveform (Fig. 10). In most muscles, the positive PCl waveform was broadly distributed across the directions for which the muscle is an agonist and has a large RMS amplitude. A negative PC1 waveform generally occurred when the muscle was mechanically antagonistic to the net force production. (Pec was a possible exception to this general rule, but we will show below that $\mathrm{BI}$ and BR conform to it.) In PD, however, the transition from a positive to a negative PCl waveform occurs within the range of directions where the RMS activity is the highest (Fig.

\section{Temporal Shift Frontal Plane}

\section{A) Posterior Deltoid}

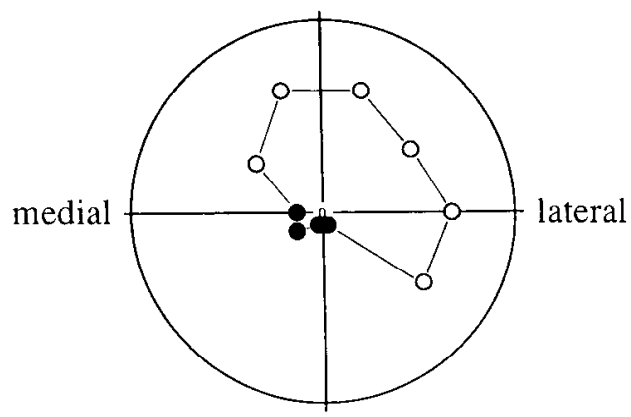

PC1

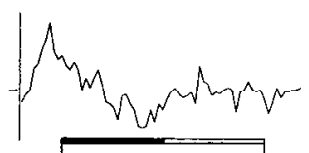

B) Medial Deltoid

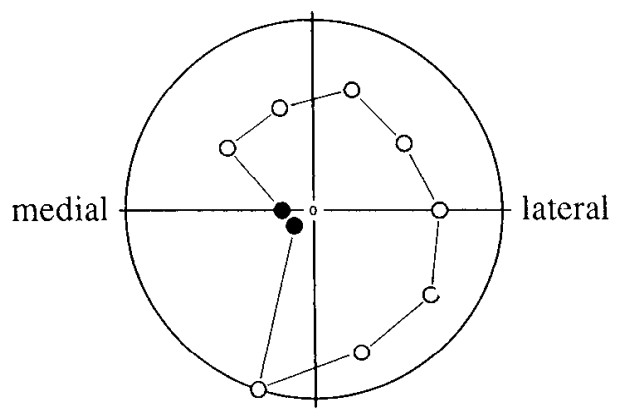

PC1 ${ }^{N} \underbrace{N}$ Mhn negative covariance
Figure 9. Temporal shift patterns. Frontal plane temporal shift plots and $\mathrm{PC1}$ waveforms for $\mathrm{PD}$, subject $\mathrm{C}(A)$, and $\mathrm{MD}$, subject $\mathrm{A}(B)$. The temporal shift scale is as in Figure 5. 


\section{Posterior Deltoid Sagittal Plane}

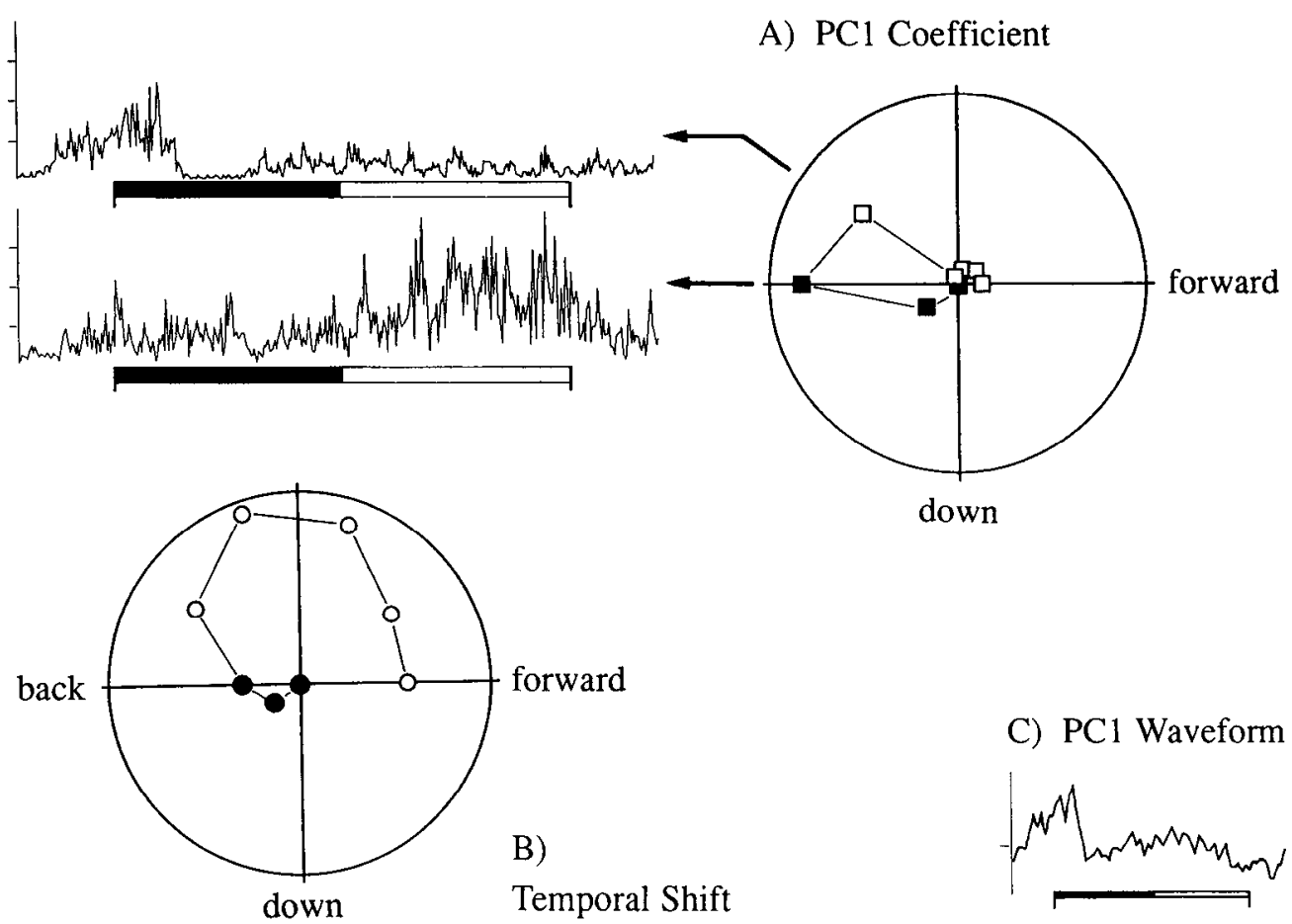

Figure 10. Transition to a reciprocal waveform. In the sagittal plane, the PD showed an abrupt transition from a positive fit to a negative fit within the region of large RMS amplitudes $(A)$. In spite of this unusual transition, the temporal shift plot $(B)$ and the $\mathrm{PCl}$ waveform $(C)$ were similar to those seen in the frontal plane for PD and in other muscles.

$10 \mathrm{~A}$ ). This is apparently due to an abrupt switch of the largest amplitude of activation from the first to the last period of the movement (as seen in the rectified, averaged EMG records). In spite of this unusually abrupt reversal, the temporal shift plot (Fig. 10B) and the PC1 waveform (Fig. 10C) conform to those shown above, for PD and MD in the frontal plane and for AD. The PCl waveform accounts for $86 \%$ of the variance.

For movement in the sagittal plane, MD does not exhibit an early burst of activation. As shown in Figure $11 \mathrm{~A}$, the $\mathrm{PCl}$ waveform exhibits a slow increase to a new level of activity: a "tonic" pattern. This is perhaps not surprising because MD is primarily useful in abduction, a lateral movement in the frontal plane, and its mechanical action in the sagittal plane is more limited. As is the case for the more phasic pattern shown in Figure $9 B$ for the frontal plane, this tonic pattern accounts for a very high percentage of the variance. The tonic waveform shown in Figure $11 \mathrm{~A}$ accounts for $89 \%$ of the variance of the set of 10 traces.

The temporal shift plot in Figure $11 \mathrm{~A}$ shows an unusual pattern. The covariance is positive in all directions, indicating an increase in activation at the end of the movement, whether the movement is backward or forward. A similar multimodal tuning for this muscle was seen in our static study (Flanders and Soechting, 1990) and suggests that MD is activated along with AD for forward movements and along with PD for backward movements. The data published in our previous study argued against the possibility that this phenomenon is due to electrical cross talk between neighboring electrodes.

\section{Biceps and brachioradialis}

In this section, we will show that the two elbow flexor muscies also exhibited a more tonic activation pattern that accounted for a high percentage of the variance (Fig. 11). For both BI and $\mathrm{BR}$, activation patterns for movements in the frontal plane were similar to those for the sagittal plane, and we will show only data from the sagittal plane. We will also contrast the tonic pattern with the more phasic pattern associated with faster movement.

Tonic patterns. Despite the fact that $\mathrm{BI}$ exhibits a tonic $\mathrm{PCl}$ waveform (Fig. 11B), the temporal shift plot follows the same basic pattern as exhibited by the deltoid. The BI crosses both the shoulder and the elbow, and its static activation reflects a compromise between its actions as a flexor at each joint (Flanders and Soechting, 1990). For movement in the sagittal plane, the activity of this muscle is indeed compromised because the shoulder is sometimes producing flexion torque while the elbow is producing extension torque (or vice versa). The EMG records for this muscle qualitatively showed more variability between subjects than for any other muscle. Nevertheless, despite these complications, the temporal shift plot shows an orderly and familiar pattern: a continuous curve in the region of positive covariance and a region of negative covariance directly opposite (cf. Figs. 6, 9, 10).

The BI PC1 waveform begins with a relatively phasic increase in activity but continues as a slow decrease from this initial activation to a new level. This waveform accounts for $91 \%$ of the variance in the set of BI traces. In the region of negative covariance (Fig. $11 \mathrm{~B}$, solid circles), the $\mathrm{PCl}$ weighting cocfficicnt is also negative, indicating a slow increase in activation to a new level (as in the MD waveform in Fig. 11 A).

For BR, the PC1 waveform showed a slow, steady transition from one level of activation to another (Fig. 1 1C). For upward/ slightly backward and upward/slightly forward movements, the transition was from an initially high level of activation to a 


\section{"Tonic" Patterns Sagittal Plane}

A) Medial Deltoid

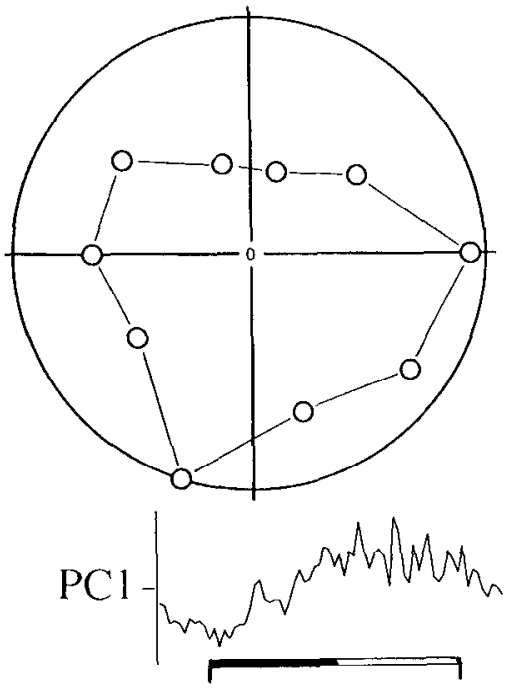

B) Biceps
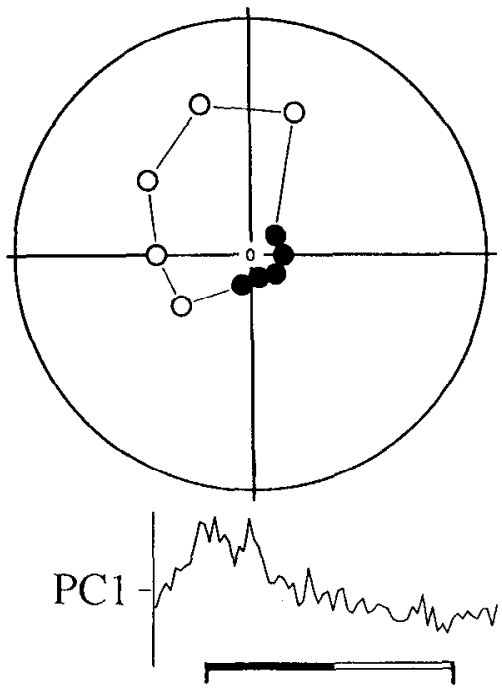

C) Brachioradialis

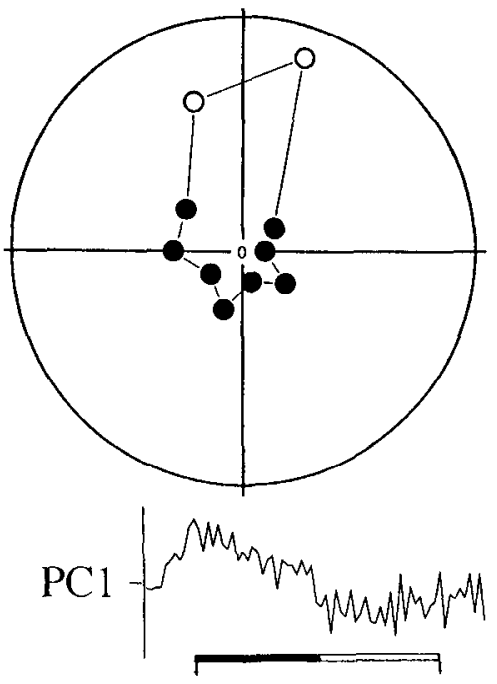

back forward negative covariance in the temporal shift

Figure 11. Three examples of sagittal plane PCl waveforms that do not show a distinct phasic portion. $A$, For MD, the temporal shift plot has no reciprocal region (subject A). $B$, For BI the temporal shift plot has a form similar to those calculated for other muscles (subject B). $C$, BR showed a steady decrease in activity for the two upward directions and a steady increase for all other directions (subject A). The scale is as in Figurc 5.

lower level (positive covariance and positive $\mathrm{PC} 1$ weighting coefficient). For all other directions, the level of activation slowly increased (as for MD, Fig. 11 $A$ ). This PCl accounted for $88 \%$ of the variance.

Phasic activation for faster movement. So far, we have presented data exclusively for moderately paced movements with a movement time of approximately $500 \mathrm{msec}$. We now compare the BR waveform from a moderately paced movement (Fig. 12, top) to the BR waveform from a movement performed as quickly as possible (Fig. 12, bottom). Figure 12, both records are five trial averages from movements upward/slightly backward in the sagittal plane. Both were recorded in the same session, and thus the electrode placement was identical. The amplitude scale is arbitrary but the same for both records. On this scale, the waveform for the moderately paced movement (top) is difficult to discern, but these same data were characterized above (in Fig. $11 C)$ with a tonic PC1 waveform. The waveform for the faster movement (bottom) is more phasic. A scaling of the time base would not be sufficient to transform the top record into the bottom record: for the faster movements, a phasic drive has come above threshold.

\section{Medial triceps}

Rectified and averaged records for MT exhibit a more obvious temporal shifting of activation onset than was exhibited by any of the other muscles (Fig. 13). Within the range of static directional tuning (Fig. 13, left), the activation onsets appear to shift

\section{Brachioradialis}
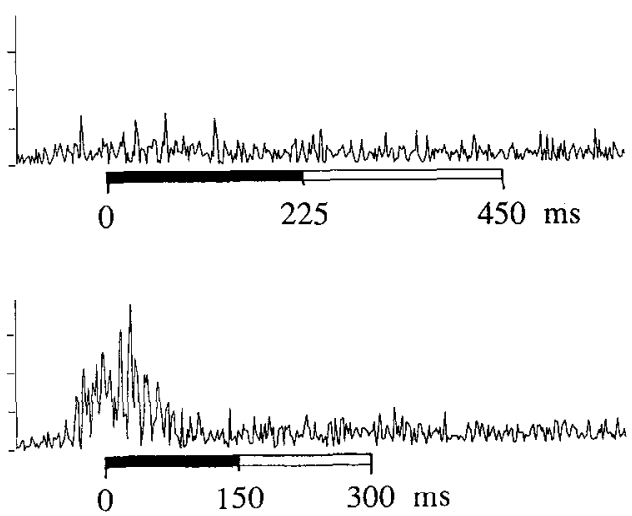

Figure 12. Increasing velocity. For faster movements, a phasic waveform appears to rise above threshold (five trial averages of rectified EMG records from subject $A$ for movement upward/slightly backward). The amplitude scale is arbitrary but the same on the top and bottom. On the $t o p$, the movement time was approximately $450 \mathrm{msec}$. On the bottom, the movement time was approximately $300 \mathrm{msec}$, and the instruction was to move as quickly as possible without overshooting the target. 
Figure 13. MT: static tuning curve (from Flanders and Soechting, 1990) and averaged, rectified EMG records for subject $A$, sagittal plane. The temporal shift in the initial activation is evident even before movement onset (black time bar). There is no apparent shift in activity in the last period of the movement (white time bar).

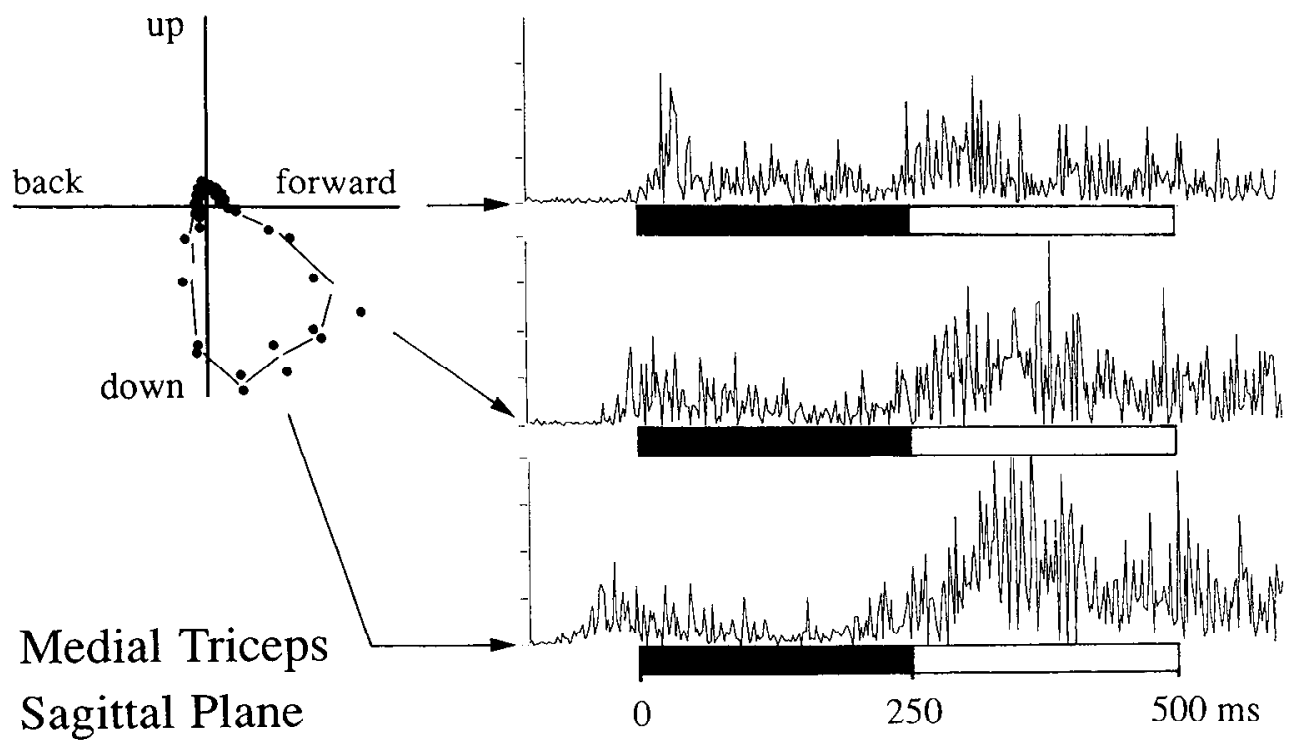

by almost $100 \mathrm{msec}$ over $72^{\circ}$ of movement direction (Fig. 13, right). The shift is apparent in the records even prior to movement onset (black time bars). Ironically, however, the covariance procedure that we used to quantify the temporal shift in other muscles indicated no temporal shift for MT in this range. This is because the activity in the last period of the record (white time bars) is relatively large and temporal shifting in this last period does not go along with the apparent shifting of the initial activation. Both our normalization and our covariance calculations were applied to the entire 700 -msec-long record. Thus, for MT, our analysis failed.

\section{Summary of temporal shifting}

To quantify the apparent shifts in initial activation in MT, we devised a reduced form of our covariance analysis and applied it to all muscles. We calculated the temporal shifts using only the first $200 \mathrm{msec}$ of each trace, $100 \mathrm{msec}$ before and $100 \mathrm{msec}$ after the movement onset. As in the analysis described above, we shifted pairs of traces in 10-msec increments as much as 100 msec earlier or later. However, for the reduced analysis, we used only positive covariance values.

One might hypothesize that a waveform of larger amplitude would come above threshold earlier. According to this hypothesis, activation onset should be proportional to the RMS amplitude: the movement direction with the largest activation would have the earliest activation, and directions on either side of this best direction would have later activations. Our data were not consistent with this simplistic hypothesis.

In Figure 14, we use a linear plot to show the amount of the temporal shift as a function of movement direction in the sagittal plane. For the four muscles that exhibited a continuous shifting function in this range, we show data from all three subjects (indicated by the solid, dashed, and dotted lines). Each data point is an average value from the two repetitions on each subject. We plot values for the direction with the largest RMS activation (zero temporal shift) and two directions on each side of this template (vertical lines delimit the ranges). For BI, the largest RMS value occurred in a different direction for one subject, and we have slightly truncated the range for clarity. If a larger RMS amplitude was always associated with an earlier activation, this would correspond to a U-shaped (upwardly concave) curve on this plot.

The MT shows a continuous unidirectional temporal shift in the range between forward and downward movements (Fig. 14, right). For forward movements, activation was later and therefore had to be shifted in the positive direction to meet the template. For downward movements, activation was earlier (cf. Fig. 13). Results from the three subjects were very similar.

Figure 14 also shows that $\mathrm{AD}$ and $\mathrm{BI}$ both exhibited unidirectional shifting functions, consistent with those shown above, on polar plots (Figs. 6, 11B). For AD, the data are consistent with those shown in Figure 6: upward movements have earlier activation, whereas downward movements have later activation. For BI, the data are consistent with those shown in Figure $11 B$ : backward movements have later activation, whereas forward movements have slightly earlier activation. Thus, for these two muscles, the reduced method gave results similar to those obtained when the analysis applied to the full-length traces.

In $\mathrm{PD}$, the temporal shifting was bidirectional around the template, giving a shifting "curve" on the linear plot in Figure 14. The orientation of this curve, however, is somewhat counterintuitive. The curve shows earlier activation on either side of the direction with the largest RMS amplitude. This phenomenon is not captured by the data shown above in Figure 10, in which traces for the downward direction were given a negative fit. It does not support a hypothetical correspondence between large amplitude activation and early activation, which would give a curve of the opposite concavity. The unidirectional shifting functions for $\mathrm{MD}, \mathrm{BI}$, and $\mathrm{AD}$ are also inconsistent with this hypothetical correspondence.

\section{Discussion}

The results show that basic waveforms of muscle activation are shifted in time for arm movements in different directions. The form of these temporal shift functions was repeatable both within and across subjects (Figs. 6, 14). The general form was also similar across many of the muscles, in spite of peculiarities in 


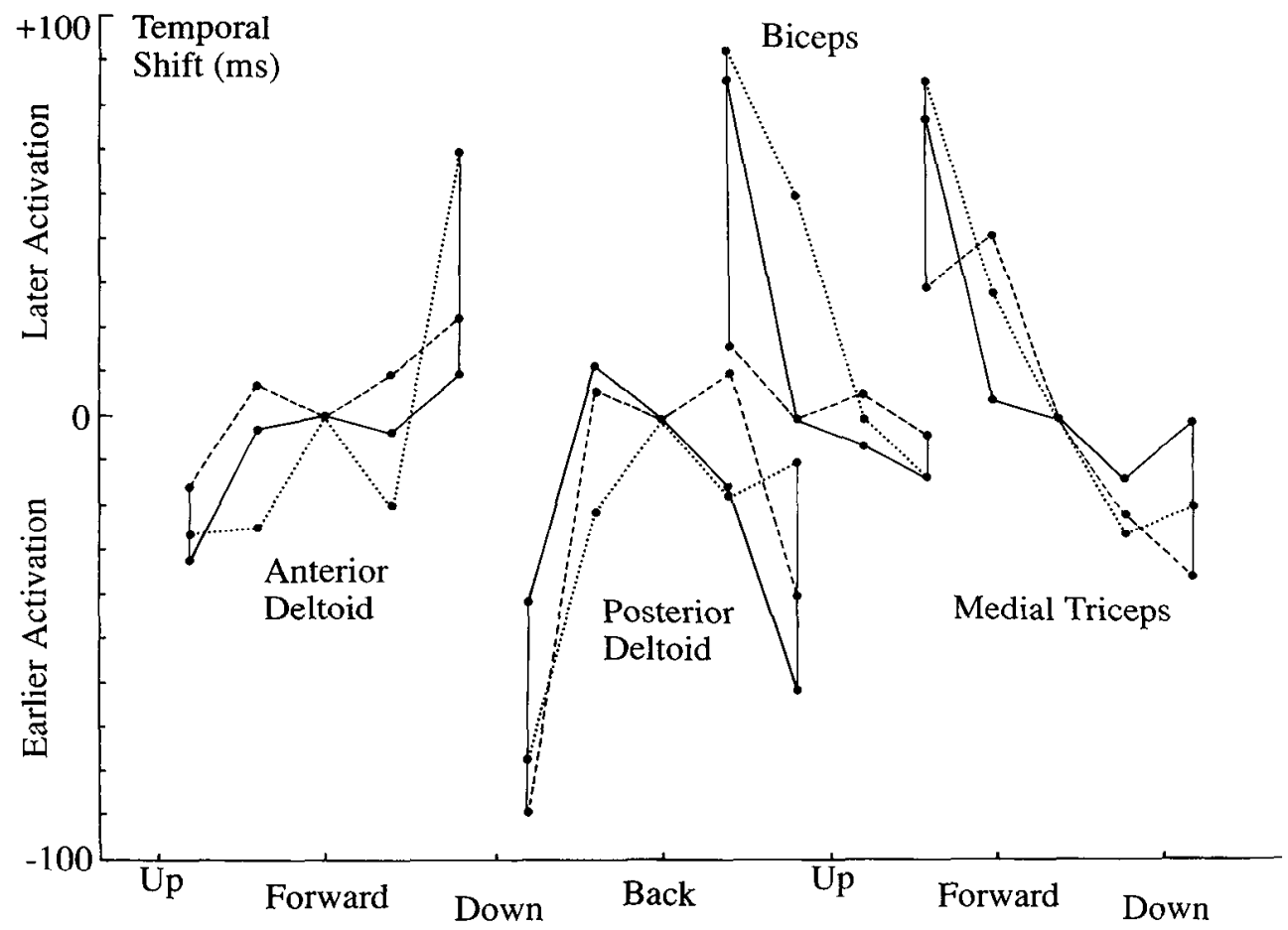

Figure 14. Summary of temporal shifting. Temporal shifts calculated using the first $200 \mathrm{msec}$ of EMG traces and using only positive covariance values. The largest RMS value (zero temporal shift) is at the center of the range of four or five directions for each muscle (the range is delimited by vertical lines). Each data point is the average of two repetitions in one subject; data from the three subjects are distinguished by solid, dashed, and dotted lines. other parameters. In our analysis of full-length traces, PD showed the usual temporal shift plot (Fig. 10B) in spite of its abrupt transition from a negative to a positive $\mathrm{PCl}$ weighting coefficient. Biceps also conformed to the usual temporal shift profile (Fig. $11 B$ ) despite the unusual mechanics of its usage and its relatively tonic waveform.

These results suggest that temporal shifting of basic waveforms is a fundamental strategy employed by the networks of neurons involved in generating arm movement. Motor patterns for movement of the hand to a target in three-dimensional space could be produced by (1) adjusting the overall amplitude according the cosine angle of the movement (Georgopoulos et al., 1984b), (2) generating a basic waveform of activation across time, and (3) delaying the waveform depending on the desired movement direction.

In the following discussion, we will examine the basic waveforms revealed by the $\mathrm{PC}$ analysis. We will compare our results concerning negative (reciprocal) waveforms and our results concerning activation onset to results from other laboratories. We will then identify some of the limitations of our experiment and the open questions for future research.

\section{Basic waveforms}

Phasic and tonic waveform. Our analysis often revealed $\mathrm{PC} 1$ waveforms with an initial curve of activation-inactivation-activation followed by a more sustained period of activation (Figs. $5 C ; 7 B ; 8 A ; 9 A, B ; 10 C)$. This waveform often accounted for over $80 \%$ of the variance in scts of 5-10 EMG traces. For lack of a better term, we have called this commonly observed PC1 waveform a "phasic and tonic" pattern.

Presumably, the phasic portion of this waveform is associated with accelerative forces, and the tonic portion is associated with a change in static posture. The PC analysis did not dissociate separate phasic and tonic components, and it is impossible to discern a point in time at which the phasic portion ends and the tonic portion begins. If phasic and tonic drives are faithfully associated with acceleration and static posture, respectively, they should temporally overlap throughout the movement. For this reason, we were reluctant to divide the waveform arbitrarily into first and last periods, and we performed most of the analyses on the entire lime segment from $100 \mathrm{msec}$ before movement onset to about $100 \mathrm{msec}$ after movement termination.

For $\mathrm{AD}$ and $\mathrm{BI}$, reducing the time window of the analysis (to the first $200 \mathrm{msec}$ ) did not significantly change the relationship between temporal shift and movement direction. For MT, however, focusing on the first $200 \mathrm{msec}$ of the EMG did change the result: from no temporal shift to the distinct temporal shift plotted in Figure 14. This could be due to the fact that MT exhibits relatively steep changes in amplitude with changes in static posture and therefore would be expected to have a tonic component with a relatively large amplitude (Fig. 13). The tonic component apparently did not shift in time and thus swamped the analysis of the full-length traces and prevented shifts in the phasic portion from being recognized. This suggests that the phasic and tonic components are separately generated drives that can be independently shifted.

A close correspondence between phasic EMG and accelerative forces has been noted by investigators of fast, targeted motor activities. For example, in their study of targeted force responses, Ghez and Gordon (1987) showed that the second derivative of force was a faithful reflection of the waveform of the agonist muscle and the inverted waveform of the antagonist muscle. In their study of the isometric arm, the (tri)phasic component was particularly evident because of the absence of the more tonic component that would have been associated with a 
change in posture. Kinesthetic input related to arm movement would be expected to enhance the phasic drive (Flanders and Cordo, 1987). However, this drive is not primarily reflexive in origin because it is also apparent in EMGs recorded in the absence of movement and in the absence of peripheral afferent input (Garland et al., 1972; Hallet et al., 1975; Rothwell et al., 1982; Sanes and Jennings, 1984). Furthermore, movement-related activity in cortical areas 4 and 5 exhibits phasic as well as tonic waveforms (Kalaska et al., 1989, 1990).

The activation pattern associated with saccadic eye movements has been termed a "pulse/slide/step" signal (reviewed by Sparks and Mays, 1990). The eye muscle EMG has a tonic (step) component associated static eye position, just as the arm muscle EMG has a tonic component related to static arm posture. The major difference between eye motor patterns and arm motor patterns is that, in arm motor patterns, a phasic drive to antagonistic muscles often serves to decelerate the movement.

Tonic waveform. For $\mathrm{MD}, \mathrm{BI}$, and $\mathrm{BR}$ in the sagittal plane, $\mathrm{PCl}$ exhibited a waveform that was apparently lacking the phasic drive (Fig. 11). This tonic waveform could account for a large amount of the variability of a set of EMG records (about $90 \%$ ). It entailed either a steady increase or decrease in activity, depending on the movement direction (Fig. $11 B, C$ ). As movement speed increased, a phasic component came above threshold (Fig. 12), suggesting that the "tonic" pattern simply lacks a phasic component rather than being completely distinct from the phasic and tonic pattern.

Faster phasic and other waveforms. We showed one example in which the PC2 contributed to the description of the directional EMG pattern (Fig. 8). In most cases, however, the higherorder PCs had a high-frequency waveform that resembled the "noise" in the EMG signal or perhaps represented the idiosyncrasies of individual trials.

\section{Reciprocal waveforms}

In addition to the temporal shifting, another robust feature of our results was the presence of negative covariance fits and negative $\mathrm{PCl}$ weighting coefficients. This usually occurred in the region directly opposite to the direction of the largest RMS amplitude. The largest RMS amplitude usually occurred in the region where the muscle was mechanically an agonist (as defined in the introductory remarks). Thus, the present study delineated regions exhibiting "agonist" and "antagonist" waveforms, just as our previous study had delimited these regions based on separable tuning curves for static force direction (Flanders and Soechting, 1990). This result is also in consonance with past work on multijoint arm movement in the horizontal plane (Wadman et al., 1980).

Wadman et al. (1980) did a systematic study of the relationship between triphasic EMG patterns and the direction of movement in the horizontal planc. The arm was supportcd against gravity, and the targeted movements were performed as quickly as possible. Thus, the EMG could be modeled as a triphasic series of pulses. For each of eight horizontal movement directions, they classified the muscle as exhibiting either "start activity" or "stop activity," based on a measurement of the EMG pulse onset. Each of the six muscles that they studied was classified as a starter or a stopper, in opposite ranges of two to four directions. Over one or two directions on each side of a start or stop range, the muscles were classified as exhibiting "support activity."

\section{Activation onset}

Wadman et al. (1980) plotted EMG onset as a function of movement direction and reported an early region where the muscle was an agonist and a later region where the muscle was an antagonist. They did not report continuous shifts within either region. This discrepancy between Wadman's data and our data may be due to the difference in our techniques of measuring the timing of activation. We based our temporal realignment measurements on the entire shape (covariance) of the above threshold waveforms. Wadman et al. (1980) measured a single point in time at which EMG came above a predetermined threshold level. We did not systematically attempt to emulate this onset measurement for the sake of comparison. However, the averaged, rectified data from AD clearly show a difference in these two techniques: in Figure 3, EMG onset (above a threshold level) roughly corresponds with RMS amplitude, a result that runs contrary to the temporal shift data shown in Figure 5 for AD (and in Figure 14 for other muscles).

Wadman et al. (1980) did show that the delay between the shoulder muscle EMG onset and the elbow muscle EMG onset was a continuous function of movement direction. Because of the unresolvable discrepancy between our techniques of measuring the timing of activation, we did not attempt to calculate shoulder-elbow data for comparison. For the same reason, we also have trouble comparing our results to those of Hasan and Karst (1989), who did a study similar to that of Wadman, based on the relative onsets of EMG above threshold.

\section{Moving onward}

Our study is predicated on the same premise as were the many past studies of the triphasic pattern associated with fast movement of a single joint (reviewed by Gottlieb et al., 1989). The premise is that a study of the relationship between EMG waveforms and kinematic parameters will lead to insight into the mechanisms of motor pattern generation. The mechanics of multijoint arm movements are significantly more complex than those of single joints because of dynamic interactions between limb segments (Hollerbach and Flash, 1982; Hoy and Zernicke, 1986; Zajac and Gordon, 1989). Although several investigators have pointed out the limitations of studies of single joint movement (see commentaries in Gottlieb et al., 1989), few have extended this approach to multijoint movements (notable exceptions are Wadman et al., 1980; Hasan and Karst, 1989). Even fewer have published EMG records for arm movement with a vertical component (an exception is Soechting and Lacquaniti, 1981). For movement in a vertical plane, an additional level of mechanical complexity arises as a result of gravitational forces (Atkeson and Hollerbach, 1985).

In our extension of past work on triphasic patterns in the horizontal plane to phasic and tonic patterns in two vertical planes, we have overlooked at least two important kinematic parameters: velocity and initial position.

Velocity. Many past investigations have revealed a relationship between movement velocity and the amplitude and duration of the agonist and antagonist bursts of the triphasic pattern (e.g., Karst and Hasan, 1987; Mustard and Lee, 1987). In Figure 12 , we showed that a phasic drive apparently appears above threshold as movement speed increases: a nonlinearity in the relationship between velocity and EMG. A more systematic study of several movement velocities is needed to document 
this phenomenon satisfactorily. In a study of movement velocity, it may be possible to dissociate phasic and tonic patterns more clearly than in this study of movement direction.

Initial position. The tonic component of the phasic and tonic pattern is presumably related to static posture. Past investigations did not reveal a significant tonic component because the movements were supported against gravity. However, the static posture of the arm is a significant determinant of activation patterns (Georgopoulos et al., 1984a; Caminiti et al., 1990; Flanders and Soechting, 1990). Kinesthetic input related to arm position may tailor muscle activation to the initial arm posture via both spinal and supraspinal mechanisms (cf. Helms Tillery et al. 1991). Further studies involving manipulation of the initial arm position may be helpful in describing the tonic component.

Clearly, further work along these lines is needed to clarify these and other issues. Another limitation of our study relates to the fact that we did a muscle-by-muscle and plane-by-plane analysis and recorded only hand kinematics. We have not yet calculated patterns across muscles or quantified the similarities and differences observed across planes, nor have we related activation to the relative kinematics of the elbow and shoulder joints. These approaches might lead to a better understanding of the overall pattern at a broader level (cf. Hasan and Karst, 1989). At a finer level, it would be interesting to know whether the reciprocal waveforms were produced by a distinct population of motor units (a possibility suggested by the work of ter Haar Romeny et al., 1982, 1984). Our study focused only on the relationship between the direction of a spatial target and the activation pattern of a given muscle. This focus, however, allowed us to identify a general strategy for the neural generation of arm muscle activation: temporal shifting of a phasic and tonic waveform.

\section{References}

Alexander GE, Crutcher MD (1990) Preparation for movement: neural representations of intended direction in three motor areas of the monkey. J Neurophysiol 64:133-150.

Atkeson CG, Hollerbach JM (1985) Kinematic features of unrestrained vertical arm movements. J Neurosci 5:2318-2330.

Caminiti R, Johnson PB, Urbano A (1990) Making arm movements within different parts of space: dynamic aspects in the primate motor cortex. J Neurosci 10:2039-2058.

Flanders M, Cordo PJ (1987) Quantification of peripherally induced reciprocal activation during voluntary muscle contraction. Electroencephalogr Clin Neurophysiol 67:389-394.

Flanders M, Soechting JF (1990) Arm muscle activation for static forces in three-dimensional space. J Neurophysiol 65:1818-1837.

Fortier PA, Kalaska JF, Smith AM (1989) Cerebellar neuronal activity related to whole arm reaching movements in the monkey. J Neurophysiol 62:198-211.

Garland H, Angel RW, Moore WE (1972) Activity of triceps brachii during voluntary elbow extension: effect of lidocain blockade of elbow flexors. Exp Neurol 37:231-235.

Georgopoulos AP, Massey JT (1988) Cognitive spatial-motor processes. 2. Information transmitted by the direction of two-dimensional arm movements and by neuronal populations in primate motor cortex and area 5. Exp Brain Res 69:315-326.

Georgopoulos AP, Caminiti R, Kalaska JF, Massey JT (1983) Spatial coding of movement: a hypothesis concerning the coding of movement direction by motor cortical populations. Exp Brain Res [Suppl] $7: 327-336$.

Georgopoulos AP, Caminiti R, Kalaska JF (1984a) Static spatial effects in motor cortex and area 5: quantitative relationships in two-dimensional space. Exp Brain Res 54:446-454.
Georgopoulos AP, Kalaska JF, Crutcher MD, Caminiti R, Massey JT (1984b) The representation of movement direction in the motor cortex: single cell and population studies. In: Dynamic aspects of neocortical function (Edelman GM, Gall WE, Cowan WM, eds), pp 501-524. New York: Wiley.

Georgopoulos AP, Kettner RE, Schwartz AB (1988) Primate motor cortex and free arm movements to visual targets in three-dimensional space. II. Coding the direction by a neuronal population. J Neurosci 8:2928-2937.

Ghez C, Gordon J (1987) Trajectory control in targeted force impulses. I. Role of opposing muscles. Exp Brain Res 67:225-240.

Gottlieb GL, Corcos DM, Agarwal GC. (1989) Strategies for the control of voluntary movements with one mechanical degree of freedom. Behav Brain Sci 12:189-250.

Hallet M, Shahani BT, Young RR (1975) EMG analysis of stereotyped voluntary movements in man. J Neurol Neurosurg Psychiatry 38: $1154-1162$.

Hasan Z, Karst GM (1989) Muscle activity for initiation of planar, two-joint arm movements in different directions. Exp Brain Res 76: 651-655.

Helms Tillery SI, Flanders M, Soechting JF (1991) A coordinate system for the synthesis of visual and kinesthetic information. J Neurosci 11:770-778.

Hollerbach JM, Flash T (1982) Dynamic interactions between limb segments during planar arm movement. Biol Cybern 44:67-77.

Hoy MG, Zernicke RF (1986) The role of intersegmental dynamics during rapid limb oscillations. J Biomech 19:867-877.

Kalaska JF, Cohen DA, Hyde ML, Prud'homme M (1989) A comparison of movement direction-related versus load direction-rclatcd activity in primate motor cortex using a two-dimensional reaching task. J Neurosci 9:2080-2102.

Kalaska JF, Cohen DA, Prud'homme M, Hyde ML (1990) Parietal area 5 neuronal activity encodes movement kinematics, not movement dynamics. Exp Brain Res 80:351-364.

Karst GM, Hasan Z (1987) Antagonist muscle activity during human forearm movements under varying kinematic and loading conditions. Exp Brain Res 67:391-401.

Mustard BE, Lee RG (1987) Relationship between EMG patterns and kinematic properties for flexion movements at the human wrist. Exp Brain Res 66:247-256.

Osborn CE, Poppele RE (1989) Components of the responses of a population of DSCT neurons determined from single unit recordings. J Neurophysiol 61:447-455.

Richmond BJ, Optican LM (1987) Temporal encoding of two-dimensional patterns by single units in the primate inferior temporal cortex. II. Quantification of response waveform. J Neurophysiol 57: 147-161.

Rothwell JC, Traub MM, Day BL, Obeso JA, Thomas PK, Marsden CD (1982) Manual motor performance in a deafferented man. Brain 105:515-542.

Sanes.IN, Iennings VA (1984) Centrally programmed patterns of muscle activity in voluntary motor behavior of humans. Exp Brain Res $54: 23-32$.

Soechting JF, Lacquaniti F (1981) Invariant characteristics of a pointing movement in man. J Neurosci 1:710-720.

Soechting JF, Lacquaniti F (1989) An assessment of the existence of muscle synergies during load perturbations and intentional movements of the human arm. Exp Brain Res 74:535-548.

Sparks DL, Mays LE (1990) Signal transformations required for the generation of saccadic eye movements. Annu Rev Neurosci 13:309336.

ter Haar Romeny BM, Denier van der Gon JJ, Gielen CCAM (1982) Changes in recruitment order of motor units in the human biceps muscle. Exp Neurol 78:360-368.

ter Haar Romeny BM, Denier van der Gon IJ, Gielen CCAM (1984) Relations between location of a motor unit in the human biceps brachii and its critical firing levels for different tasks. Exp Neurol 85: 631-650.

Wadman WJ, Denier van der Gon JJ, Derksen RJA (1980) Muscle activation patterns for fast goal-directed arm movements. J Hum Move Stud 6:19-37.

Zajac FE, Gordon ME (1989) Determining muscle's force and action in multi-articular movement. Exerc Sport Sci Rev 17:187-230. 